\title{
[Letter to the Editor] \\ Incorrect assignment of affected nucleotides in footprinting/probing experiments
}

Probing the structure of nucleic acids complexed with proteins or drugs using different enzymes and/or nucleic acid cleavage/modification reagents is a valuable technique for studying interactions of nucleic acids with their binding partners. This approach has been successfully used in the analysis of nucleic acid-protein interactions $(1,2)$, chromatin structure (3), and the binding of small molecules to DNA/RNA (4). These existing protocols rely on precise assignment of digestion/modification patterns to the sequence of interest at the singlenucleotide level. Lately, tremendous success has been achieved in developing high-throughput approaches to analyze nucleic acid digestion/modification patterns for studying DNA/RNA structure in living cells. Despite this, primer extension-based methods (5) are still used to identify the nucleotide position of the cleavage/modification by comparison with dideoxy sequencing extensions initiated from the same primer and loaded on the same gel (6).

Here, we report that the use of the standard dideoxy sequencing protocol utilizing dITP instead of dGTP may cause misidentification of the position of a modified nucleotide in primer extension assays. dITP is widely used in sequencing reactions to reduce secondary structure formation and prevent base compression (7). However, incorporation of inosine influences the electrophoretic properties of DNA, which, as we show here, can lead to misinterpretation of the modification pattern.

We have studied the secondary structure of the $18 \mathrm{~S}$ region in the pre-ribosomal precursor RNA using the selective 2'-hydroxyl acylation and primer extension (SHAPE) method with the reagent 1-methyl-7-nitroisatoic anhydride (1M7) (\#POI-129-50, Prime
Organics, Inc., Woburn, MA), which selectively reacts with flexible RNA nucleotides at the ribose 2'-hydroxyl group (8). Sites of modification were detected due to their ability to halt primer extension. A control primer extension reaction, with RNA not exposed to $1 \mathrm{M} 7$ [only to the solvent, DMSO (\#D8418, Sigma-Aldrich, St. Louis, MO)], and dideoxy sequencing reactions were run in parallel. Note that reverse transcription stops one nucleotide prior to the position of the modified nucleotide. We discovered that one of the sites of modification, a hyper-reactive nucleotide, was repeatedly attributed to different but successive positions in the 18S rRNA (Figure 1). Careful inspection of the experimental conditions revealed a single deviation in the protocol that initially escaped our attention, namely, the use of dITP in the sequencing reactions in some experiments.

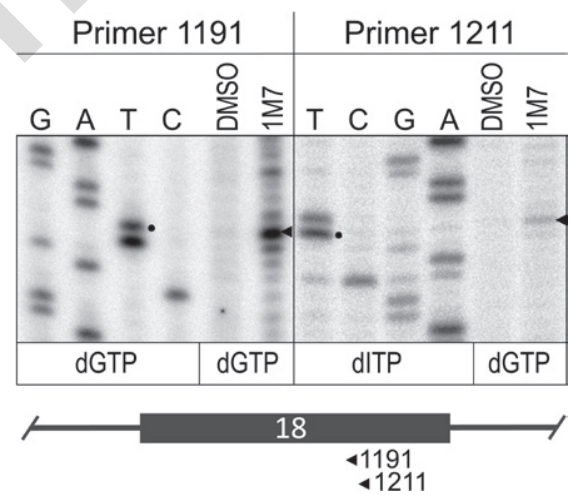

Figure 1. SHAPE analyses of nucleotide reactivities established by primer extension and resolved by denaturing PAGE together with sequencing ladders synthesized using dGTP or dITP, as indicated. The positions of the two primers (1191 and 1211) are shown in the schematic beneath the gel image. A black arrowhead indicates a primer extension stop caused by a SHAPE hyperreactive nucleotide. The co-migrating band in each of the two sequencing ladders is indicated by a black dot. Note the ambiguity in relating the primer extension band to the dITP and dGTP ladders.
To clarify this issue, we synthesized sequencing ladders using either the dGTP or the dITP provided with the Sequenase Version 2.0 DNA sequencing kit (\#707701KT, Thermo Fisher Scientific, Waltham, MA) and loaded these ladders on the same gel with primer extension products containing dGTP from 1M7-modified

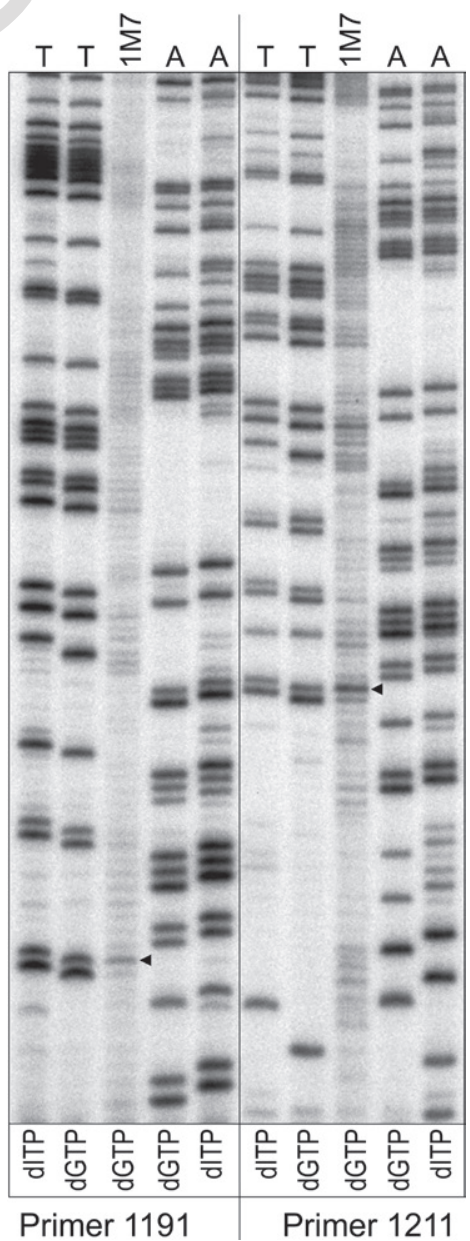

Figure 2. SHAPE analysis of nucleotide reactivity by primer extension using dGTP. Sequencing ladders for A and T, synthesized using dGTP or dITP and resolved on the same gel, show the migration differences for dGTP and dITP. All loading mixtures were equalized in terms of volume and salt/buffer conditions Arrowhead indicates the same SHAPE band shown in Figure 1. 
RNA obtained with the same primer (Figure 2). All samples had identical loading volumes and salt/buffer conditions. We also used two different primers to ensure that the observed phenomenon was not primer-specific (see Figures 1 and 2). The results clearly demonstrate a mobility shift between the dITP and dGTP samples, with slower mobility in the dITPrelative to dGTP-containing sample lanes. This result was in accordance with our previous data (Figure 1), and explained the difficulty in correctly relating the primer extension bands to the sequencing ladder.

We believe that this problem could also occur when using capillary electrophoresis rather than conventional slab systems, since both methods have identical modus operandi. It should be noted that dITP has been used instead of dGTP in reverse transcriptase-driven primer extension in SHAPE analysis (as well as in the accompanying dideoxy sequencing ladders) to reduce band compressions near $\mathrm{G}$ nucleotides $(9,10)$. However, in accordance with previous observations of the inefficiency of dIMP incorporation during reverse transcription (11), this also resulted in misinterpretation of the modification pattern at $\mathrm{C}$ nucleotides, due to reverse transcriptase pausing. Thus, correct sequence attribution of the cleavage/modification pattern in primer extension-based nucleic acid probing experiments requires use of either only dGTP or only dITP in the dNTP mixes for both the sequencing and the primer extension reactions.

\section{Author contributions}

S.B. and F. L. performed experiments. S.B., F.L., and L.W. analyzed the data. S.B., F.L., and L.W. prepared the manuscript.

\section{Acknowledgments}

This work was supported by the Swedish Research Council (grant 621-2010-5236) and by Carl Tryggers Stiftelse, project number CTS 15:531, granted to L.W. We would like to thank BioTechniques editors for bringing our attention to important publications relevant to this study.

\section{Competing interests}

The authors declare no competing interests.

\section{References}

1. Dey, B., S. Thukral, S. Krishnan, M. Chakrobarty, S. Gupta, C. Manghani, and V. Rani. 2012. DNA-protein interactions: Methods for detection and analysis. Mol. Cell. Biochem. 365:279-299.

2. McHugh, C.A., P. Russell, and M. Guttman. 2014. Methods for comprehensive experimental identification of RNA-protein interactions. Genome Biol. 15:203.

3. Belikov, S., C. Åstrand, and Ö. Wrange. 2009. FoxA1 Binding Directs Chromatin Structure and the Functional Response of a Glucocorticoid Receptor-Regulated Promoter. Mol. Cell. Biol. 29:5413-5425.

4. Dervan, P.B. 2001. Molecular Recognition of DNA by Small Molecules. Bioorg. Med. Chem. 9:2215-2235.

5. Carey, M.F., C.L. Peterson, and S.T. Smale. 2013. The primer extension assay. Cold Spring Harb. Protoc. 2013:164-173.

6. van Nues, R.W. and N.J. Watkins. 2017. Unusual C /D motifs enable box C/D snoRNPs to modify multiple sites in the same rRNA target region. Nucleic Acids Res. 45:2016-2028.

7. Hirao, I., Y. Nishimura, Y. Tagawa, K. Watanabe, and K. Miura. 1992. Extraordinarily stable mini-hairpins: electrophoretical and thermal properties of the various sequence variants of $d(G C G A A A G C)$ and their effect on DNA sequencing. Nucleic Acids Res. 20:3891-3896.

8. Merino, E.J., K.A. Wilkinson, J.L. Coughlan, and K.M. Weeks. 2005 RNA structure analysis at single nucleotide resolution by selective 2'-hydroxyl acylation and primer extension (SHAPE). J. Am. Chem. Soc. 127:4223-4231.

9. Kladwang, W., C.C. VanLang, P. Cordero, and R. Das. 2011. Understanding the errors of SHAPE-directed RNA structure modeling. Biochemistry 50:8049-8056.

10. Mortimer, S.A. and K.M. Weeks. 2009. Time-resolved RNA SHAPE chemistry: quantitative RNA structure analysis in one second snapshots and at single nucleotide resolution. Nat. Protoc. 4:1413-1421.

11. Auer, T., J.J. Sninsky, D.H. Gelfand, and T.W. Myers. 1996. Selective amplification of RNA utilizing the nucleotide analog dITP and Thermus thermophilus DNA polymerase. Nucleic Acids Res. 24:5021-5025.

Sergey Belikov*, Fredrik Lackmann, and Lars Wieslander* Department of Molecular Biosciences, The Wenner-Gren Institute, Stockholm University, Stockholm, Sweden

BioTechniques 63:105-106 (September 2017) doi 10.2144/000114585

Keywords: primer extension; Sanger sequencing; dITP

Received 30 May 2017; accepted 19 July 2017.

Address correspondence to Sergey Belikov or Lars Wieslander, Department of Molecular Biosciences, The Wenner-Gren Institute, Stockholm University, SE-106 91 Stockholm, Sweden. E-mail: sergey.belikov@su.se or lars.wieslander@su.se

To purchase reprints of this article, contact: biotechniques@fosterprinting.com 